\title{
Influência da Temperatura e da Natureza do Catalisador na Polimerização do Glicerol
}

\author{
Miguel de A. Medeiros \\ Departamento de Ciências Biológicas, UFT \\ Jeob de C. Rezende, Maria H. Araújo, Rochel M. Lago \\ Departamento de Química, UFMG
}

\begin{abstract}
Resumo: Neste trabalho, a polimerização do glicerol na presença de catalisador ácido $\left(\mathrm{H}_{2} \mathrm{SO}_{4}\right.$ ou $\left.\mathrm{H}_{3} \mathrm{PO}_{4}\right)$ e básico $(\mathrm{NaOH})$, para produzir resinas termofixas foi investigada. Os resultados mostraram que as variáveis como a temperatura de reação, o tipo e a concentração do catalisador são críticas para a obtenção de bons rendimentos e seletividade para materiais poliméricos. Em condições otimizadas, i.e. $\mathrm{H}_{2} \mathrm{SO}_{4}$ como catalisador, $140{ }^{\circ} \mathrm{C}$ por 24 horas, foi possível obter polímeros com $98 \%$ de seletividade. Após polimerização, os materiais obtidos foram submetidos a extrações com diferentes solventes, i.e. água, THF e hexano. Os extratos foram caracterizados por ESI(+)-MS e por espectroscopia na região do infravermelho, que permitiram verificar a presença de oligômeros de até seis unidades monoméricas. Para menores tempos de reação, e.g. 4 horas, foi possível obter elevada seletividade (100\%) para oligômeros, mas com baixa conversão de glicerol (25\%). Além disso, verificou-se que os outros catalisadores $\left(\mathrm{H}_{3} \mathrm{PO}_{4}\right.$ e $\left.\mathrm{NaOH}\right)$ apresentam baixa atividade para promover a polimerização do glicerol, e.g. rendimento de $25 \%$ após 24 horas.
\end{abstract}

Palavras-chave: Glicerol, polimerização, resina termofixa, poliglicerol.

\section{Influence of Temperature and Nature of the Catalyst on Glycerol Polymerization}

Abstract: In this work, an investigation was made of the glycerol polymerization in the presence of acid $\left(\mathrm{H}_{2} \mathrm{SO}_{4}\right.$ or $\left.\mathrm{H}_{3} \mathrm{PO}_{4}\right)$ and base $(\mathrm{NaOH})$ catalysts to produce thermosetting resins. The results showed that the reaction temperature and catalyst concentration are critical to obtain good yield and selectivity. Under optimum condition, i.e. $\mathrm{H} 2 \mathrm{SO} 4$ catalyst, $140{ }^{\circ} \mathrm{C}$ for 24 hours it was possible to obtain polymers with $98 \%$ selectivity. After the polymerization the materials were submitted to extractions with different solvents, i.e. water, THF and hexane. The extracts were characterized by ESI(+)-MS (Electro-Spray Ionization Mass Spectrometry) and by FTIR, showing the presence of short-chain oligomers (up to six units). For shorter reaction times, e.g. 4 hours, it was possible to obtain high selectivity (100\%) for oligomers, but with low glycerol conversion (25\%). It was also observed that the other catalysts $\left(\mathrm{H}_{3} \mathrm{PO}_{4}\right.$ and $\left.\mathrm{NaOH}\right)$ showed low activity to promote glycerol polymerization, e.g. yields of $25 \%$ after 24 hours.

Keywords: Glycerol, polymerization, thermosetting resin, polyglycerol.

\section{Introdução}

A crescente produção de biodiesel, através da transesterificação ${ }^{[1-3]}$ de óleos vegetais ou gorduras animais (Figura 1), em todo o mundo ${ }^{[4]}$ e em especial no Brasil, deve levar a um aumento significativo do volume de glicerol no mercado.

Para cada tonelada de triglicerídeos processada, são produzidos pelo menos $100 \mathrm{~kg}$ de glicerol ${ }^{[4-6]}$. Como consequência, o preço do glicerol cai fortemente no mercado em todo o mundo ${ }^{[7]}$. Essa queda de preço é uma tendência mundial, não sendo diferente no Brasil, que em 2005 registrou preços de glicerol em torno de $\mathrm{R} \$ 3000 \mathrm{t}^{-1}$, mas já em 2007, a mesma quantidade de glicerol era comercializada por $\mathrm{R} \$ 1700 \mathrm{t}^{-1[8]}$. E nas regiões próximas às usinas de biodiesel, em 2009, o preço não ultrapassou $\mathrm{R} \$ 700 \mathrm{t}^{-1[8]}$. Diante dessa situação, torna-se estratégico o desenvolvimento de novos processos para a conversão de glicerol em produtos de maior valor agregado $^{[9]}$, pois a produção e mercado de glicerol, derivado da indústria petroquímica, já são estáveis.

Atualmente, o glicerol derivado da indústria petroquímica (que apresenta alta pureza) é empregado na produção de $\operatorname{cosméticos}^{[10,11]}$, fármacos $^{[12]}$, bronzeadores ${ }^{[13]}$, aditivos alimentares ${ }^{[10,11]}$ e estabilizante para $\mathrm{PVC}^{[14]}$, aplicações que não são facilmente acessíveis para o glicerol derivado do biodiesel (que apresenta grau de pureza variável ${ }^{[15]}$.
Uma alternativa de transformação química, entre diversas descritas na literatura ${ }^{[15-25]}$, para consumir parte desse glicerol derivado do biodiesel é a reação de polimerização (Figura 2), pois os polímeros produzidos podem ter diferentes aplicações, tais como substitutos de polióis, e.g. álcool polivinílico ${ }^{[26]}$, aditivos para tintas ${ }^{[10]}$, lubrificantes ${ }^{[27]}$, aditivos para cimentos ${ }^{[4,28]}$ e matéria-prima para produção de poliuretanas e resinas ${ }^{[12]}$ de aplicações pouco nobres.

Na reação de polimerização, as unidades de glicerol se condensam através dos grupos hidroxilas, formando éteres, que podem ter cadeias carbônicas de comprimento e complexidade variada. Vários trabalhos ${ }^{[27,29-36]}$ descrevem a polimerização do glicerol para obter oligômeros (Figura 2), ( $\leq \mathrm{n} \leq 18)$, utilizando catalisadores homogêneos e heterogêneos. Nesses trabalhos, são apresentadas moléculas de cadeia curta e simples (di, tri, tetra, penta e hexaglicerol) como produto. No entanto, a polimerização para a formação de resinas termofixas derivadas da polimerização extensiva do glicerol (Figura 2), $(\mathrm{n} \geq 19)$, foi pouco estudada até o momento ${ }^{[37]}$.

\section{Experimental}

Glicerol P.A. (11 mmol), $\mathrm{H}_{2} \mathrm{SO}_{4}, \mathrm{H}_{3} \mathrm{PO}_{4}$ ou $\mathrm{NaOH}$, em concentrações variando entre 0,5 e $10 \mathrm{~mol} \%$, foram misturados em um reator de vidro de $200 \mathrm{~mL}$. O sistema foi aquecido a 140, 180 ou $220^{\circ} \mathrm{C}$, por até 24 horas. Durante todo o período de reação, o sistema foi mantido sob vácuo. 


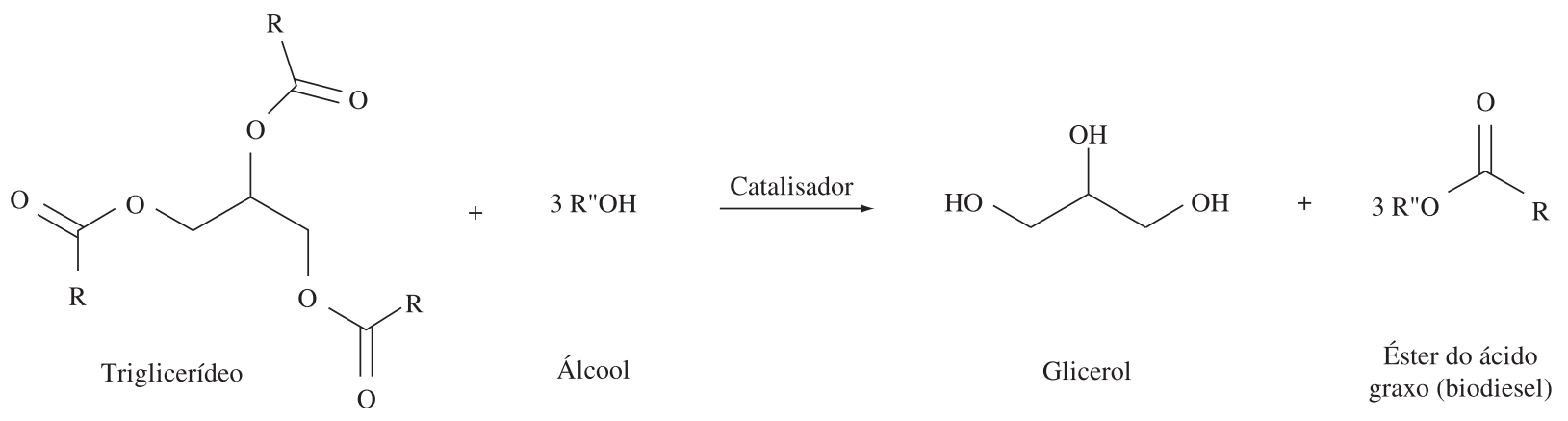

Figura 1. Produção de biodiesel, através da transesterificação de triglicerídeo.<smiles>OCC(O)CO</smiles>

Glicerol

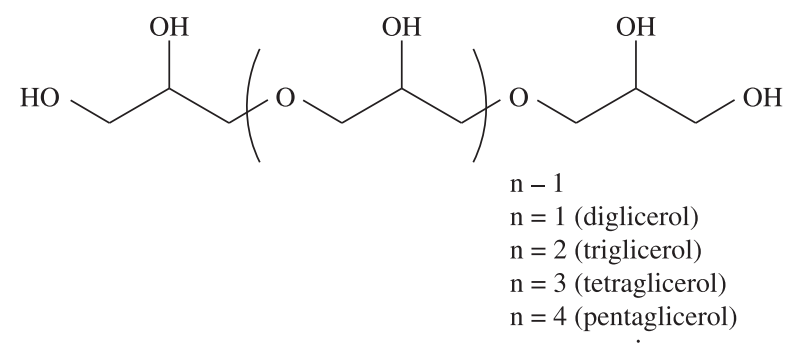

Figura 2. Polimerização do glicerol.

As reações foram monitoradas por análise termogravimétrica em uma termobalança Shimadzu TGA60H, em fluxo de nitrogênio $(100 \mathrm{~mL} / \mathrm{min})$, com taxa de aquecimento de $10^{\circ} \mathrm{C} / \mathrm{min}$. Os produtos foram caracterizados também por (i) espectroscopia vibracional na região do infravermelho em um equipamento Perkin Elmer Spectrum GX FT-IR System, na região de 4000 a $650 \mathrm{~cm}^{-1}$, utilizando o método de reflectância - ATR, (ii) microscopia eletrônica de varredura em um equipamento JEOL-84A, (iii) análise elementar em um equipamento Perkin Elmer PE 2400 CHN Elemental Analyzer, (iv) análise térmica por calorimetria diferencial de varredura (DSC), em um equipamento Shimadzu DSC50 (atmosfera inerte de $\mathrm{N}_{2}$ e razão de aquecimento de $10{ }^{\circ} \mathrm{C} / \mathrm{min}$ ) e (v) ESI(+)-MS - Espectrometria de Massas com Ionização por Electrospray, em um equipamento Agilent-1100, em modo positivo.

As amostras de resinas foram submetidas a sucessivas lavagens com solventes em refluxo, por 4 horas, na seguinte ordem: hexano, tetrahidrofurano (THF) e água. Após a lavagem, verificaram-se nos solventes, através de ESI(+)-MS, a presença de glicerol, oligômeros e seus produtos de desidratação.

\section{Resultados e Discussão}

Neste trabalho foram investigadas duas diferentes variáveis na polimerização do glicerol: (i) efeito da temperatura e (ii) influência do catalisador.

\section{(i) Efeito da temperatura}

A polimerização do glicerol foi realizada na presença de $3 \mathrm{~mol} \%$ de $\mathrm{H}_{2} \mathrm{SO}_{4}$, em 4 horas de reação, em diferentes temperaturas $\left(140,180\right.$ e $\left.220{ }^{\circ} \mathrm{C}\right)$. As amostras foram nomeadas com códigos, e.g. S3T4-140, onde S3 indica a concentração de catalisador $\mathrm{H}_{2} \mathrm{SO}_{4}$ ( $\mathrm{S} 3=3 \mathrm{~mol} \%$ de $\mathrm{H}_{2} \mathrm{SO}_{4}$ ), T4 indica o tempo de reação ( $\mathrm{T} 4=4$ horas de reação) e 140 indica a temperatura do sistema.

A polimerização catalítica do glicerol, em diferentes temperaturas, foi acompanhada utilizando análise termogravimétrica. A partir da análise dos resultados, foi possível identificar a fração glicerol convertida, seja em oligômero ou em polímero, presente nas amostras (Figura 3).
A análise das curvas apresentadas na Figura 3 indica que o experimento realizado a $140{ }^{\circ} \mathrm{C}$ (S3T4-140), produz pequena quantidade de oligômeros/polímero (25\%), até mesmo após 4 horas de reação. Já os experimentos realizados a 180 e $220{ }^{\circ} \mathrm{C}$ (S3T4-180 e S3T4-220) produz grande quantidade de oligômeros/polímero (95\%), desde a segunda hora de reação. Após a terceira hora de reação, $100 \%$ da massa do sistema (S3T4-180 e S3T4-220) correspondem a produtos de condensação do glicerol (oligômeros ou polímero). Esses resultados indicam que a elevação de temperatura é extremamente favorável para a maior atividade do sistema de oligomerização/polimerização do glicerol, mostrando que não há diferença, em rendimentos, para os sistemas a 180 e $220{ }^{\circ} \mathrm{C}$, pois ambos são muito ativos e convertem totalmente o glicerol presente no meio (em 4 horas de reação).

Para quantificar o teor de oligômeros e também de polímero, presente nas amostras, foram realizadas extrações consecutivas com hexano, THF e água, solventes de polaridade distintas e capazes de dissolver apenas oligômeros e derivados. O material extraído, constituído basicamente de oligômeros, foi pesado e caracterizado por ESI(+)-MS. Através dos dados de ESI(+)-MS e do teor de material dissolvido nos solventes, pode-se calcular a seletividade para oligômeros e polímero presentes nas amostras S3T4-140, S3T4-180 e S3T4-220 (Tabela 1).

Ao analisar cada uma das amostras (S3T4-140, S3T4-180 e S3T4220), observou-se que $140^{\circ} \mathrm{C}$ é uma temperatura favorável para produzir oligômeros (di, tri, tetra e pentaglicerol) e seus produtos de desidratação, caracterizados no espectro ESI(+)MS apresentado na Figura 4 (os sinais $m / z 57$ e $75 ; m / z 131$ e $149 ; m / z, 205$ e $223 ; m / z, 281$ e 297 e $m / z, 371$ são os íons derivados dos produtos de desidratação do glicerol, di, tri, tetra e pentaglicerol, respectivamente).

Na temperatura de $180{ }^{\circ} \mathrm{C}(\mathrm{S} 3 \mathrm{~T} 4-180)$ é obtido um material com seletividade de $82 \%$ para polímeros e apenas $18 \%$ para oligômeros e derivados, com taxa de conversão de $90 \%$. Já a $220{ }^{\circ} \mathrm{C}$ (S3T4-220), observa-se total conversão do glicerol presente na amostra em produtos de oligomerização/polimerização. A seletividade para o material S3T4-220 ficou assim distribuída: 91\% para polímeros e apenas 9\% para oligômeros e derivados. Esses dois materiais (S3T4-180 e S3T4-220) foram então analisados por Análise 


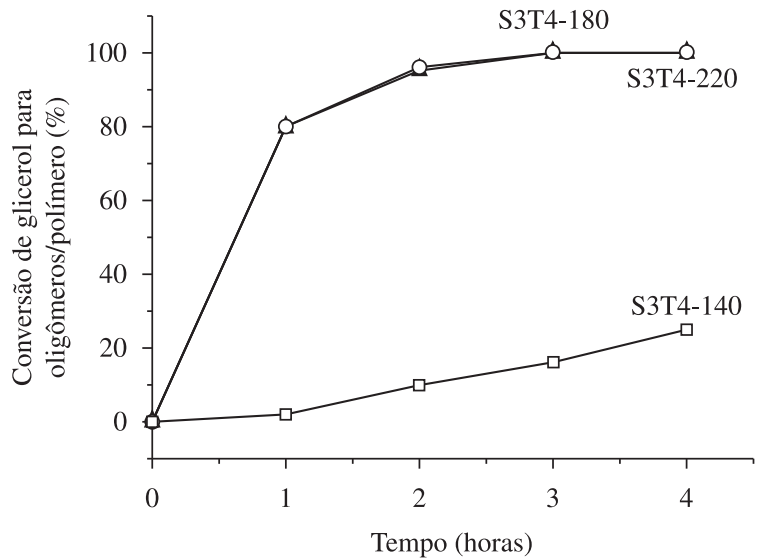

Figura 3. Formação de oligômeros/polímero, a 140 (S3T4-140), 180 (S3T4-180) e $220^{\circ} \mathrm{C}$ (S3T4-220), em função do tempo.

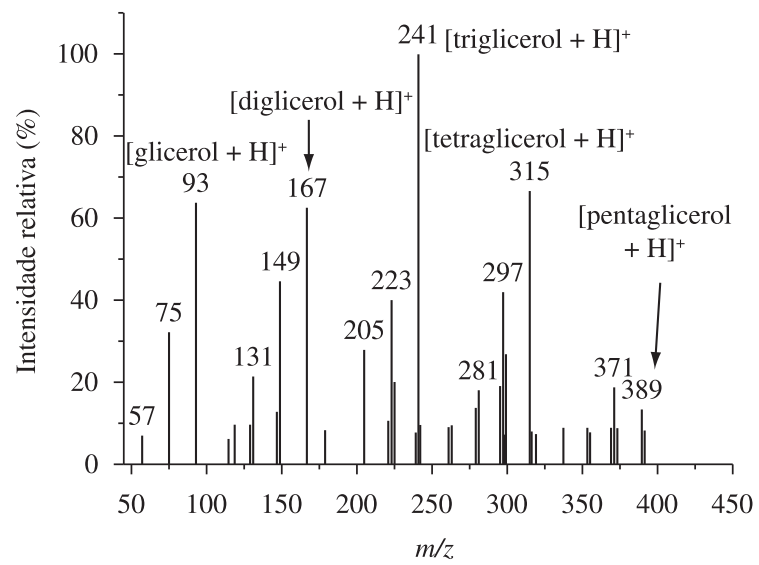

Figura 4. Espectro ESI(+)-MS da amostra S3T4-140, mostrando os sinais $(\mathrm{M}+1)$ do glicerol e dos oligômeros.

Elementar (Tabela 2), que mostrou significativa redução da \% $\mathrm{H}$ $\% \mathrm{O}$ com aumento da \% em relação ao glicerol. Esses resultados indicam a perda de moléculas de $\mathrm{H}_{2} \mathrm{O}$ e a crescente reticulação da cadeia carbônica. A partir desses resultados é possível determinar a fórmula mínima de cada resina (S3T4-180 e S3T4-220), (Tabela 2), que evidencia o aumento do teor de carbono e redução do teor de hidrogênio e oxigênio.

Análise térmica por calorimetria diferencial de varredura (DSC) para as amostras S3T4-180 e S3T4-220 não mostraram eventos atribuídos à fusão ou à transição vítrea $(\mathrm{Tg})$, indicando que os materiais não sofrem fusão, nem se tornam maleáveis ao serem aquecidos até $300{ }^{\circ} \mathrm{C}$, quando se inicia a decomposição térmica dos materiais. Esses resultados sugerem que as cadeias do polímero (nas duas amostras, S3T4-180 e S3T4-220) apresentam grande quantidade de ligações cruzadas, o que dificulta a mobilidade das cadeias, não permitindo que os materiais se tornem maleáveis ou sofram fusão.

Para verificar grupos funcionais presentes na estrutura dos materiaisS3T4-140,S3T4-180eS3T4-220, realizaram-seanálisespor Espectroscopia Vibracional na Região do Infravermelho (Figura 5). Observou-se que a elevação da temperatura $\left(140-220^{\circ} \mathrm{C}\right)$ influencia na diminuição das intensidades relativas dos grupos característicos do glicerol e oligômeros: bandas de estiramento $\mathrm{OH}\left(3386 \mathrm{~cm}^{-1}\right)$, de ligação C-O de álcool secundário simétrico $\left(1215 \mathrm{~cm}^{-1}\right)$ e de ligação C-O de álcool primário $\left(1045 \mathrm{~cm}^{-1}\right)$. É possível observar ainda a presença de uma forte banda em $1735 \mathrm{~cm}^{-1}$ (que surge nas amostras S3T4-180 e S3T4-220) atribuída a grupos carbonilas, identificadas

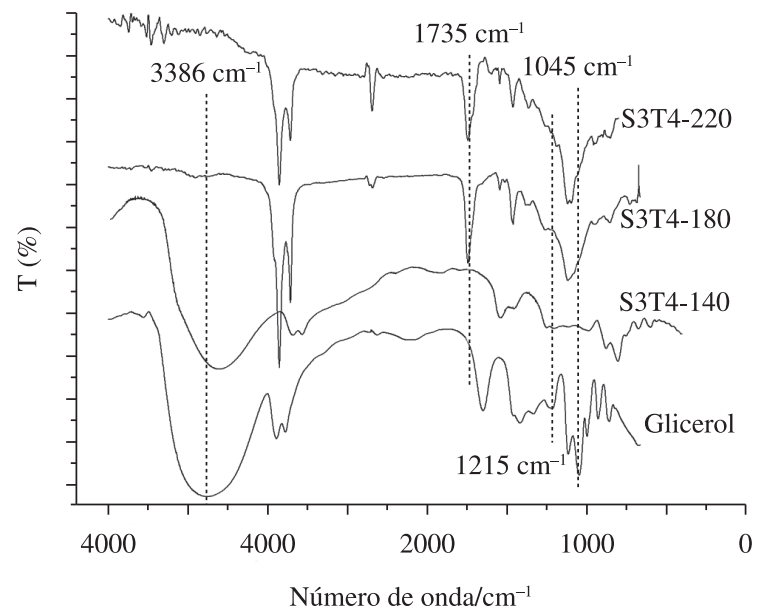

Figura 5. Espectros de Infravermelho das amostras de glicerol, S3T4-140, S3T4-180 e S3T4-220.

Tabela 1. Variação da temperatura na polimerização do glicerol ${ }^{a}$.

\begin{tabular}{ccccc}
\hline Amostras & $\begin{array}{c}\text { Temp. } \\
\left({ }^{\circ} \mathbf{C}\right)\end{array}$ & $\begin{array}{c}\text { Conv. } \\
(\%)\end{array}$ & \multicolumn{2}{c}{ Seletividade } \\
\cline { 4 - 5 } & & & $\begin{array}{c}\text { Oligômeros e } \\
\text { derivados }^{\mathbf{b}}\end{array}$ & Polímeros \\
\hline S3T4-140 & 140 & 25 & 100 & 0 \\
S3T4-180 & 180 & 90 & 18 & 82 \\
S3T4-220 & 220 & 100 & 9 & 91 \\
\hline
\end{tabular}

${ }^{a}$ Condições de reação: glicerol $=11 \mathrm{mmol} ; \mathrm{H}_{2} \mathrm{SO}_{4}=0,3 \mathrm{mmol}$; $\mathrm{t}=4$ horas. ${ }^{b}$ Produtos da condensação e desidratação.

Tabela 2. Dados de Análise Elementar do glicerol e das amostras de resina (S3T4-180 e S3T4-220).

\begin{tabular}{ccccc}
\hline Amostras & $\mathbf{C}(\%) *$ & $\mathbf{H}(\%) *$ & $\mathbf{O}(\%) *$ & Fórmula mínima \\
\hline Glicerol & 39,1 & 8,7 & 52,2 & $\mathrm{C}_{3} \mathrm{H}_{8} \mathrm{O}_{3}$ \\
S3T4-180 & 58,0 & 7,1 & 34,8 & $\mathrm{C}_{5} \mathrm{H}_{7} \mathrm{O}_{2}$ \\
S3T4-220 & 59,0 & 5,6 & 35,5 & $\mathrm{C}_{5} \mathrm{H}_{6} \mathrm{O}_{2}$ \\
\hline
\end{tabular}

* percentagem de cada elemento presente na amostra.

como pertencentes a aldeídos, principalmente, acroleína (composto formado a partir da desidratação do glicerol - perda de 2 moléculas de $\mathrm{H}_{2} \mathrm{O}$ - em meio ácido e em aquecimento a altas temperaturas ${ }^{[38]}$ ).

As resinas S3T4-180 e S3T4-220 também foram analisadas por Microscopia Eletrônica de Varredura (MEV) (Figura 6), que permitiu observar as principais formas de estruturação do material polimérico, em nível microscópico. As imagens exibidas na Figura 6a, b e c mostram, em corte longitudinal, estruturas esféricas que podem ser bolhas oriundas da liberação extensiva de vapor de água, durante a reação de polimerização. As superfícies dessas bolhas e também a superfície do material, apresentaram ondulações, que são características da redução de volume do polímero, logo após liberar vapor de água, quando o polímero ainda era maleável (antes do enrijecimento) (Figura 6c e d).

Em nível macroscópico, as resinas S3T4-180 e S3T4-220, se apresentam na forma de um polímero rígido, com brilho plástico e coloração preta.

\section{(ii) Efeito do catalisador e de sua concentração}

Com o objetivo de verificar a ação do catalisador no processo de oligomerização/polimerização do glicerol, selecionou-se a temperatura de $140{ }^{\circ} \mathrm{C}$ para o estudo da influência do catalisador. Inicialmente, foi verificado o efeito da natureza do catalisador, utilizando 3 mol\% de $\mathrm{H}_{2} \mathrm{SO}_{4}$ (S3T4-140), $\mathrm{H}_{3} \mathrm{PO}_{4}$ (P3T4-140) e $\mathrm{NaOH}$ (B3T4-140), conforme é apresentado na Figura 7. 


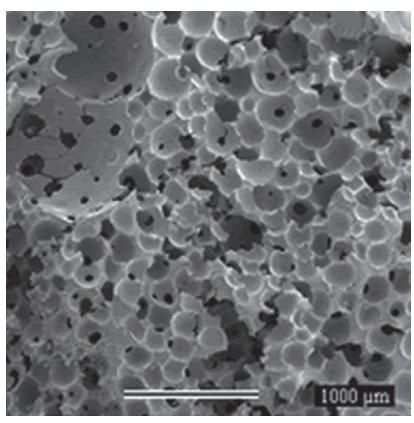

(a)

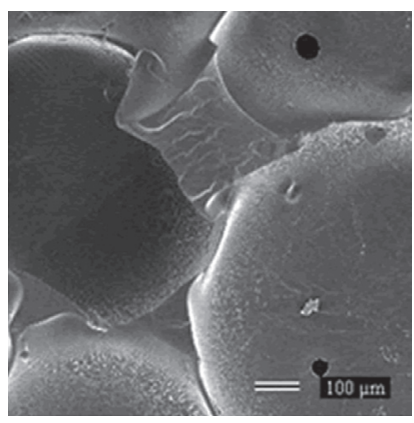

(b)

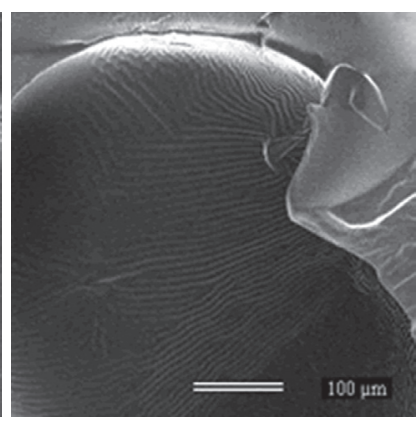

(c)

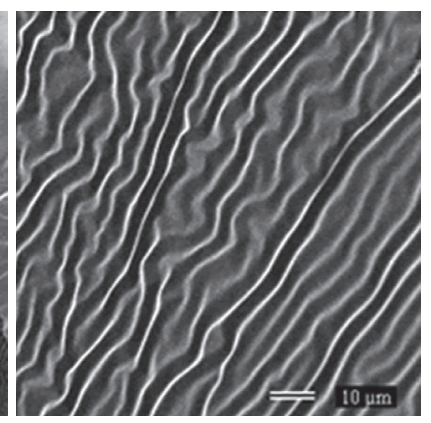

(d)

Figura 6. Imagens de MEV das resinas (S3T4-180 e S3T4-220).

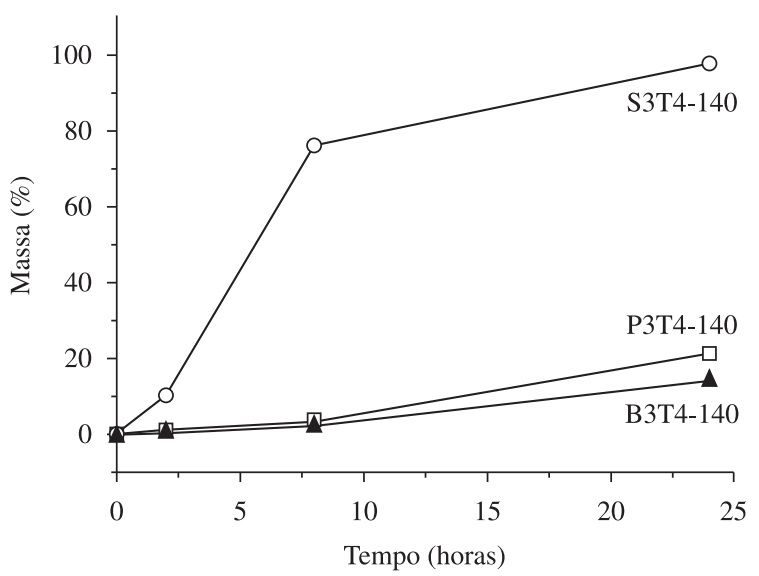

Figura 7. Comparativo entre diferentes catalisadores (S3T4-140: $\mathrm{H}_{2} \mathrm{SO}_{4}$, P3T4-140: $\mathrm{H}_{3} \mathrm{PO}_{4}$ e B3T4-140: NaOH) da oligomerização/polimerização do glicerol, a $140{ }^{\circ} \mathrm{C}$.

Os sistemas promovidos por $\mathrm{H}_{3} \mathrm{PO}_{4}(\mathrm{P} 3 \mathrm{~T} 4-140)$ e $\mathrm{NaOH}$ (B3T4-140) apresentam baixa conversão em oligômeros/polímeros (14 - NaOH e 21\% - $\mathrm{H}_{3} \mathrm{PO}_{4}$ ), mesmo após 24 horas de reação. Já o sistema promovido por $\mathrm{H}_{2} \mathrm{SO}_{4}$, desde a segunda hora de reação apresenta considerável formação de oligômeros/polímeros (11\%), aumentando rapidamente ao longo do processo (76\% em 6 horas), atingindo $98 \%$ em 24 horas.

A polimerização do glicerol é baseada na formação de éteres a partir da condensação entre duas (ou mais) moléculas (ou grupos $\mathrm{OH}$ ) de álcool. E um dos mecanismos mais favoráveis para a formação de éteres é através da protonação de um grupo álcool ( $\mathrm{ROH} 2$ ), seguida da condensação de outro álcool e liberação de uma molécula de água ${ }^{[39,40]}$. Dessa maneira, acredita-se que $\mathrm{oH}_{2} \mathrm{SO}_{4}$ protona com maior facilidade os grupos alcoóis, pois é um ácido relativamente forte, quando comparado com $\mathrm{H}_{3} \mathrm{PO}_{4}$ e NaOH.

\section{Variação da concentração de $\mathrm{H}_{2} \mathrm{SO}_{4}$}

A polimerização do glicerol foi realizada a $140{ }^{\circ} \mathrm{C}$, variando a concentração $(0,5 ; 1 ; 3$ e $5 \mathrm{~mol} \%)$ do catalisador $\mathrm{H}_{2} \mathrm{SO}_{4}$. Para acompanhar o processo de polimerização nas diferentes concentrações de catalisador, foram realizadas medidas de viscosidade da solução reacional a cada 15 minutos.

É conhecido que a polimerização catalítica do glicerol é um processo que pode alterar significativamente a viscosidade da solução, ao ponto de se formar um polímero reticulado, alongando a cadeia em duas dimensões, horizontal e vertical.

Como mecanismo para entender como é a variação da viscosidade, a partir da polimerização do glicerol, realizaram-se testes de viscosidade relativa (viscosidade da solução / viscosidade do glicerol) para amostras de reação com 0,$5 ; 1 ; 3$ e 5 mol\% de $\mathrm{H}_{2} \mathrm{SO}_{4}$, durante os 60 minutos iniciais de reação (Figura 8).

As curvas apresentadas na Figura 8 indicam um aumento expressivo da viscosidade da solução, ao variar o percentual em mol do catalisador de 0,5-5\%. Entretanto, esse aumento é gradativo, na medida em que se eleva a concentração de $\mathrm{H}_{2} \mathrm{SO}_{4}$. É interessante observar que o sistema promovido por $5 \mathrm{~mol} \%$ de catalisador é bastante ativo, pois foram necessários apenas 45 minutos para produzir um polímero sólido (não foi possível medir a viscosidade, pois o material enrijeceu), enquanto que nos outros sistemas foram necessários pelo menos 120 minutos.

Os sistemas promovidos por 0,$5 ; 1$ e 3 mol\% de $\mathrm{H}_{2} \mathrm{SO}_{4}$ mostraram perfis de curva de viscosidade semelhantes (Figura 8), mas com inclinações variáveis (quanto maior a concentração do catalisador, maior a inclinação da curva de viscosidade). Com o aumento da concentração de $\mathrm{H}_{2} \mathrm{SO}_{4}$ (0,5-3\%) é esperado maior número de condensações simultâneas, consequentemente, a seletividade do catalisador para grupos hidroxilas reativos do glicerol diminui, originando estruturas complexas, que oferecem aumento de viscosidade à solução (em 60 minutos: sistema promovido por $0,5 \%$ $\rightarrow$ viscosidade relativa de $4 ; 1 \% \rightarrow$ viscosidade relativa de 51 e $3 \%$ $\rightarrow$ viscosidade relativa de 169 vezes a do glicerol). Nos 15 minutos iniciais de reação, a viscosidade do meio praticamente não se altera. Acredita-se que durante esse período, esteja ocorrendo a formação de oligômeros lineares e seus produtos de desidratação. Entretanto, à medida que a reação de polimerização avança, as cadeias carbônicas dos éteres formados se tornam maiores e mais complexas, devido principalmente a formação de ramificações e algumas ligações entre cadeias paralelas de oligômeros e/ou polímeros. E é esse aumento do tamanho e complexidade das cadeias dos éteres formados que dá à solução, crescente viscosidade, atingindo 169 vezes a viscosidade do glicerol em apenas 60 minutos (sistema promovido por $3 \mathrm{~mol} \%$ de $\mathrm{H}_{2} \mathrm{SO}_{4}$ ), pois a movimentação das cadeias fica cada vez mais difícil.

\section{Variação da concentração de $\mathrm{H}_{3} \mathrm{PO}_{4}$}

Os resultados observados através de análise termogravimétrica (Figura 7) mostraram que o ácido sulfúrico é um catalisador mais efetivo para a polimerização do glicerol do que o ácido fosfórico, em concentração de $3 \mathrm{~mol} \%$. Acredita-se que esse resultado seja explicado pela baixa dissociação do $\mathrm{H}_{3} \mathrm{PO}_{4}$ em solução de glicerol, o que não deve ocorrer com o $\mathrm{H}_{2} \mathrm{SO}_{4}$. Dessa maneira, variou-se a concentração do $\mathrm{H}_{3} \mathrm{PO}_{4}(3,5$ e $10 \mathrm{~mol} \%)$ com o objetivo de compreender a ação desse catalisador na polimerização do glicerol. Os resultados desses experimentos estão apresentados na Figura 9.

A análise do gráfico apresentado na Figura 9 mostra que a elevação da concentração de catalisador $\mathrm{H}_{3} \mathrm{PO}_{4}$ favorece a oligomerização/ polimerização do glicerol. Entretanto, mesmo utilizando $5 \mathrm{~mol} \%$ de $\mathrm{H}_{3} \mathrm{PO}_{4}$, é observado rendimento de apenas $21 \%$ para oligômeros e ao elevar a concentração para $10 \mathrm{~mol} \%$, passa-se a observar a presença de polímero ( $12 \%$ da amostra), mas ainda há pequena quantidade de 


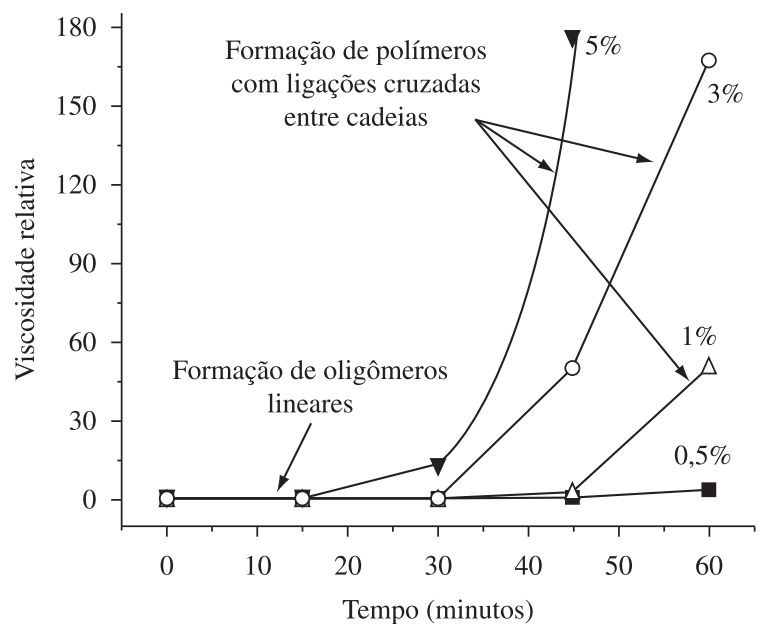

Figura 8. Variação da viscosidade relativa da solução, para a reação de polimerização do glicerol, com 0,$5 ; 1 ; 3$ e $5 \mathrm{~mol} \%$ de $\mathrm{H}_{2} \mathrm{SO}_{4}$ (os valores de viscosidade são relativos ao do glicerol).

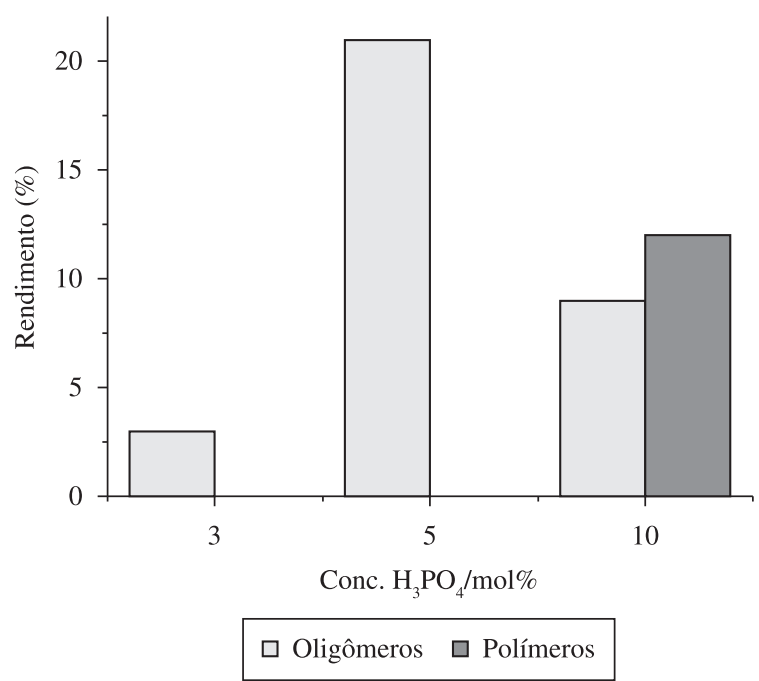

Figura 9. Rendimento para os oligômeros e polímeros, de acordo com a concentração de catalisador $\mathrm{H}_{3} \mathrm{PO}_{4}$. Condições de reação: glicerol $=11 \mathrm{mmol}$, $\mathrm{T}=140{ }^{\circ} \mathrm{C}$ e $\mathrm{t}=8$ horas. Dados obtidos através de análise termogravimétrica.

oligômeros, apenas 9\%. Esses resultados mostram que a elevação da concentração de $\mathrm{H}_{3} \mathrm{PO}_{4}$ favorece o processo de oligomerização/ polimerização do glicerol, mas o aumento de rendimento para polímero ou oligômero não é significativo, quando comparado com o $\mathrm{H}_{2} \mathrm{SO}_{4}$.

A partir dessa série de experimentos, variando a concentração de $\mathrm{H}_{2} \mathrm{SO}_{4}$ e $\mathrm{H}_{3} \mathrm{PO}_{4}$, é possível verificar que o processo de polimerização do glicerol é grandemente favorecido por catalisadores fortemente ácidos, como $\mathrm{H}_{2} \mathrm{SO}_{4}$. E a utilização de ácidos fracos e bases fortes se mostraram de pouca expressão para produzir oligômeros e/ou polímeros, com grandes rendimentos.

\section{Conclusões}

A oligomerização/polimerização do glicerol ocorre em sistema catalisado por ácidos e bases, em temperaturas iguais ou superiores a $140{ }^{\circ} \mathrm{C}$, com tempo de reação de até 24 horas. Entretanto, o processo é grandemente favorecido por catalisadores fortemente ácidos (tal como o $\left.\mathrm{H}_{2} \mathrm{SO}_{4}\right)$ e pouco promovido por catalisador básico $(\mathrm{NaOH})$ ou ácido fraco $\left(\mathrm{H}_{3} \mathrm{PO}_{4}\right)$.
A maior conversão de glicerol (100\%) em oligômeros ou polímeros é atingida com o emprego de $3 \mathrm{~mol} \%$ de $\mathrm{H}_{2} \mathrm{SO}_{4}$ e $220{ }^{\circ} \mathrm{C}$, em 4 horas de reação. Nessas condições, há seletividade de $91 \%$ para polímeros e apenas $9 \%$ para oligômeros. Já nas mesmas condições de reação, mas utilizando $140{ }^{\circ} \mathrm{C}$ como temperatura do sistema, observa-se seletividade de $100 \%$ para oligômeros, com $25 \%$ de conversão de glicerol. Empregando concentração de catalisador $\left(\mathrm{H}_{2} \mathrm{SO}_{4}\right)$ maior, por exemplo, $5 \mathrm{~mol} \%$, é possível observar a formação de polímero sólido em apenas 45 minutos de reação. Já em concentrações menores $(0,5 ; 1$ e $3 \mathrm{~mol} \%)$, é observada variação gradativa de viscosidade, que atinge 169 vezes a viscosidade do glicerol (quando se utiliza $3 \mathrm{~mol} \%$ de $\mathrm{H}_{2} \mathrm{SO}_{4}$ ), em apenas 60 minutos.

A Espectrometria de Massas com Ionização por Electrospray se mostrou uma técnica importante para acompanhar a fase inicial da polimerização do glicerol, quando se observaram a formação de oligômeros (di, tri, tetra e pentaglicerol) e seus produtos de desidratação.

\section{Agradecimentos}

CNPq, FAPEMIG, CAPES e UFMG.

\section{Referências Bibliográficas}

1. Rinaldi, R.; Garcia, C.; Marciniuk, L. L.; Rossi, A. V. \& Schuchardt, U. - Quim. Nova, 30, p.1374 (2007).

2. Geris, R.; Santos, N. A. C. dos; Amaral, B. A.; Maia, I. S.; Castro, V. D. \& Carvalho, R. M. - Quim. Nova, 30, p.1369 (2007).

3. Suarez, P. A. Z. \& Meneguetti, S. M. P. - Quim. Nova, 30, p.2068 (2007).

4. Pagliaro, M. \& Rossi, M. - "The Future of Glycerol: New uses of a versatile raw material", RSC Publishing, Cambridge (2008).

5. Karinen, R. S. \& Krause, A. O. I. - Appl. Catal. A: Gen., 306, p.128 (2006).

6. Dasari, M. A.; Kiatsimkul, P. P.; Sutterlin, W. R. \& Suppes, G. J. - Appl. Catal. A: Gen., 281, p.225 (2005).

7. Behr, A.; Eilting, J.; Irawadi, K.; Leschinski, J. \& Lindner, F. - Green Chem., 10, p.13 (2008).

8. Jornal Valor Econômico, http://www.valoronline.com.br/ valoreconomico/285/empresasetecnologia/empresas/Glicerina+de+bi odiesel+inunda+mercado+no+pais+e+derruba+precos+,,,51,4289003 html, acessado em: 14 de fevereiro de 2008.

9. Klepacova, K.; Mravec, D.; Hajekova, E. \& Bajus, M. - Petroleum and Coal, 45, p.54 (2003).

10. Lemke, D. W. U.S. Patent 6620 904, 2003. - Chem. Abstr., 136, p.370466 (2003).

11. Mota, C. J. A.; da Silva, C. X. A. \& Gonçalves, V. L. C. - Quim. Nova, 32, p.639 (2009).

12. Werpy, T. \& Petersen, G. Top Value Added Chemicals From biomass, U.S. Department of Energy, Oak Ridge, Richland, (2004).

13. Kimura, H. - Appl. Catal. A: Gen., 105, p.147 (1993).

14. Kumar, T. N.; Sastry, Y. S. R. \& Lakshminarayama, G. - J. Am. Oil Chem. Soc., 66 (1989).

15. Medeiros, M. A.; Oliveira, D. L.; Sansiviero, M. T. C.; Araujo, M. H. \& Lago, R. M. - J Chem Technol Biotechnol (in press, DOI 10.1002/ jctb.2309)

16. Silva, C. X. A. da; Gonçalves, V. L. C. \& Mota, C. J. A. - Green Chem, 11, p.38 (2009).

17. Silva, C. R. B. da; Gonçalves, V. L. C.; Lachter, E. R. \& Mota, C. J. A. - J. Braz. Chem. Soc., 20, p.201 (2009).

18. Cho, G. H. P.; Yeong, S. K.; Ool, T. L. \& Chuah, C. H. - J. Surf. Deter., 9, p.147 (2006). 
19. Diaz, I.; Marquez-Alvarez, C.; Mohino, F.; Pérez-Pariente, J. \& Sastre, E. - J. Catal., 193, p.295 (2000).

20. Ramaya, S.; Brittain, A.; de Almeida, C.; Mok, W. \& Antal, M. J. Fuel, 66, p.1363 (1987).

21. Atia, H.; Armbruster, U. \& Martin, A. - J. Catal., 258, p.71 (2008).

22. Luna, F. J. \& Schuchardt, U. - Quim. Nova, 24, p.885 (2001).

23. Huber, G. W.; Shabaker, J. W. \& Dumesic, J. A. - Science, 300, p.2075 (2003).

24. Stein, Y. S.; Antal, M. J. J. \& Jones, M. - J. Anal. and Appl. Pyrolysis, 4, p.283 (1983).

25. Medeiros, M. A.; Sansiviero, M. T. C.; Araujo, M. H. \& Lago, R. M. Appl. Clay Sci., 45, p.213 (2009).

26. Medeiros, M. A.; Oliveira, L. C. A.; Gonçalves, M.; Araújo, M. H. \& Lago, R. M. Anais do $21^{\circ}$ Simposio Iberoamericano de Catálisis, Málaga - ES (2008).

27. Barrault, J.; Jerome, F. \& Pouilloux, Y. Lipid Technol., 17, p.131 (2005).

28. Moorer, H. H. \& Anderegg, C. M. US Patent US4204877 (1980).

29. Kraft, A. U.S. Patent 6649 690, 2003. Chem. Abstr., 139, p.7661 (2005).

30. Eshuis, J. I.; Laan, J. A. \& Potman, R. P. U.S. Patent 5635588 (1997).

31. Jeromin, L.; Gutsche, B.; Bunte, R. \& Jordan, V. U.S. Patent 5710 350, 1998. Chem Abstr., 121,p.230325 (1998).
32. Klepácová, K.; Miravec, E. \& Bajus, M. - Chem. Pap., 60, p.224 (2006)

33. Lemke, D. W. U.S. Patent 2002058 781, 2002; Chem. Abstr., 136, p.370466 (2002)

34. Barrault, J.; Clacens, Y. \& Pouilloux, Y. Topics Catal., 27, p.137 (2004).

35. Márquez-Alvarez, C.; Sastre, E. \& Pérez-Pariente, J. Topics Catal., 27, p.105 (2004).

36. Medeiros, M. A.; Araújo, M. H.; Augusti, R.; Oliveira, L. C. A. \& Lago, R. M. - J. Braz. Chem. Soc., 20, p.1667 (2009).

37. Medeiros, M. A.; Rezende, J. C.; Araújo, M. H. \& Lago, R. M. Anais do $10^{\circ}$ Congresso Brasileiro de Polímeros, Foz do Iguaçu- BR (2009).

38. Watanabe, T.; Iida, Y.; Aizawa, T.; Ainda, M. \& Inomata, H. - Bioresour. Technol., 98, p.1285 (2007).

39. Solomons, T. W. G. - "Química Orgânica”, LTC, Rio de Janeiro (1996).

40. Smith, M. \& March, J. - "Advanced organic chemistry: reactions, mechanisms, and structure", John Wiley \& Sons, New York (2001).

Enviado: 16/11/09

Reenviado: $30 / 01 / 10$

Aceito: 04/03/10

DOI: $10.1590 /$ S0104-14282010005000033 